\title{
Bioestabilização anaeróbia de resíduos sólidos orgânicos em reatores de batelada
}

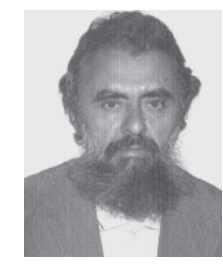

Valderi D. Leite ${ }^{1}$, Wilton S. Lopes² \& Shiva Prasad ${ }^{3}$

\author{
1 UEPB. R. Vigário Calixto, 1475, Catolé, CEP 58104-485, Campina Grande, PB. E-mail: valderi@paqtc.rpp.br (Foto) \\ 2 PRODEMA/UFPB/UEPB. R. Papa João Paulo II, 325, Nova Brasilia, CEP 58103-600, Campina Grande, PB. Fone: (83) 322-5008. \\ E-mail: wslopes@bol.com.br \\ 3 UFPB. R. Aprígio Veloso, 882, Bodocongó, CEP 58109-970, Campina Grande, PB. Fone: (83) 333-3171
}

Protocolo $096-14 / 08 / 2000$

\begin{abstract}
Resumo: O processo de bioestabilização anaeróbio pode ser empregado para o tratamento de uma grande variedade de substratos, dentre os quais pode-se destacar os resíduos sólidos orgânicos. Neste trabalho, o processo de bioestabilização anaeróbio foi utilizado, quando do tratamento de resíduos sólidos orgânicos putrescíveis de origem urbana e rural. Os resíduos de origem urbana eram constituídos, basicamente, de restos de fruta, verduras e folhagens, enquanto o rúmen bovino era o resíduo rural utilizado. As proporções de rúmen empregadas foram de 5, 10 e $15 \%$ (percentagem em peso).O trabalho foi realizado em um sistema experimental, constituído basicamente por quatro reatores anaeróbios de batelada, com capacidade unitária de 20 L. A eficiência do processo foi determinada tomando-se, como parâmetros avaliativos, os sólidos totais voláteis (STV), a demanda química de oxigênio (DQO) e o nitrogênio total Kjedhal (NTK). Salienta-se que este processo de tratamento bioestabiliza os resíduos orgânicos putrescíveis, tornando-os biodisponíveis de aplicação nos solos, além de produzir metano, que pode ser utilizado como fonte alternativa de energia para fins domésticos e industriais. A análise dos dados deste trabalho demonstra que o desempenho do processo de bioestabilização foi função do percentual de rúmen utilizado.
\end{abstract}

Palavras-chave: resíduos orgânicos, anaeróbio, reator de batelada

\section{Anaerobic biostabilization of organic solid waste in batch reactors}

\begin{abstract}
The anaerobic biostabilization process can be employed for the treatment of a large variety of substrates, among which the organic solid waste can be cited. In this work, the anaerobic biostabilization process was employed to treat the putrescible organic solid waste of urban and rural origin. The urban origin waste basically consisted of fruits peelings, vegetables and foliage, whereas the rural solid waste was only the bovine rumen. The proportions of rumen used were 5, 10 and $15 \%$ (weight basis). The work was carried out on an experimental scale, basically consisting of four anaerobic batch reactors, each with a capacity of $20 \mathrm{~L}$. The efficiency of the process was determined by evaluation of the following parameters: total volatile solids (TVS), chemical oxygen demand (COD) and total Kjedhal nitrogen (TKN). It may be pointed out that this treatment process biostabilizes the putrescible organic waste, making it available for application in the soils as well as producing of methane, which can be used as a source of alternative energy for domestic and industrial purposes. The analysis of the data shows that the performance of the biostabilization process is a function of the percentage of rumen used.
\end{abstract}

Key words: organic waste, anaerobic, batch reactor

\section{INTRODUÇ̃̃O}

Os resíduos sólidos urbanos são constituídos pelos resíduos sólidos domiciliares e comerciais, resíduos de varrição e serviços, em cuja composição gravimétrica as frações mais representativas são formadas por restos de alimentos, papel, papelão e plástico.

Atualmente, constata-se que, na maioria dos países, principalmente nos desenvolvidos ou em estágio de desenvolvimento, a problemática associada aos resíduos sólidos vem-se agravando acentuadamente, em decorrência de vários fatores, dentre os quais se pode citar: o acentuado crescimento demográfico dos centros urbanos, criação e/ou mudanças de hábitos, elevado nível de vida, ajustada prioritariamente pelo elevado índice de concentração de renda de pequena parte da população, desenvolvimento industrial e a política de globalização, priorizando sobretudo o aspecto formal da economia de mercado (Leite, 1998).

No Brasil são produzidas cerca de $100.000 \mathrm{t} \mathrm{d}^{-1}$ de resíduos sólidos urbanos, sendo que apenas $10 \%$ deste quantitativo 
recebem tratamento e/ou disposição final adequada (IPT, 2000).

Dos resíduos sólidos urbanos produzidos a nível nacional, um percentual médio de 55\% (percentagem em peso) é de matéria orgânica putrescível, passível de fermentação (IPT, 2000). Portanto, um quantitativo em torno de $50.000 \mathrm{t} \mathrm{d}^{-1}$ de matéria orgânica putrescível é lançado de maneira irregular, gerando impactos ambientais de diversas magnitudes, razão por que se torna necessário o desenvolvimento de alternativas tecnológicas que objetivem o aproveitamento racional desta matéria orgânica, propiciando a redução dos impactos ambientais e a melhoria da qualidade de vida da população, principalmente da população mais carente.

Nesta contextualização, a utilização do processo anaeróbio desponta como alternativa promissora, haja vista, biodisponibilizar uma grande variedade de resíduos orgânicos putrescíveis, produzindo composto com características essenciais de agente condicionador de solo e um representativo potencial energético, advindo do gás metano.

A principal desvantagem associada ao uso de digestores anaeróbios para o tratamento de resíduos sólidos, refere-se ao longo tempo necessário para bioestabilização do material (Metcalf \& Eddy, 1991). Vários estudos têm sido realizados objetivando-se aumentar a taxa de bioestabilização da matéria orgânica e, em sua maioria, lodo de esgoto sanitário tem sido utilizado como inóculo e os resultados têm mostrado a viabilidade de aplicação do processo.

O processo de tratamento anaeróbio consiste na decomposição de resíduos orgânicos putrescíveis na ausência de oxigênio livre, tendo como produtos finais $\mathrm{CH}_{4}, \mathrm{CO}_{2}, \mathrm{NH}_{3}$, quantidade traços de outros gases e ácidos orgânicos de baixo peso molecular (Polprasert, 1989).

Segundo Leite (1997) o tratamento anaeróbio da fração orgânica putrescível dos resíduos sólidos urbanos inoculada com lodo de esgoto sanitário, apresentou taxa média de produção de $\mathrm{CH}_{4}$ de $0,25 \mathrm{~N} \mathrm{~m}^{3} \mathrm{~kg}^{-1} \mathrm{DQO}_{\text {aplicada }}$, o que corresponde a uma per capita de produção de metano em torno de $17 \mathrm{NLCH}_{4}$ $\mathrm{hab}^{-1} \mathrm{~kg}^{-1}$.

Objetivou-se, com o presente trabalho, avaliar a influência do rúmen bovino, utilizado como inóculo, no processo de bioestabilização anaeróbia da fração orgânica putrescível dos resíduos sólidos urbanos, em reatores de batelada operando com alta concentração de sólidos.

\section{MATERIAL E MÉTODOS}

O sistema experimental foi instalado e monitorado nas dependências do Laboratório de Saneamento Ambiental do Centro de Ciências e Tecnologia da UEPB, no período de janeiro de 1998 a janeiro de 1999.

A fração orgânica putrescível dos resíduos sólidos urbanos utilizada no trabalho experimental, foi coletada da EMPASA (Empresa Paraibana de Alimentos e Serviços Agrícolas) unidade de Campina Grande, PB. Estes resíduos eram constituídos basicamente de restos de fruta e verduras advindos do descarte de parte dos produtos comercializados na EMPASA. O rúmen bovino foi conseguido em matadouros particulares da cidade de Campina Grande, PB.
Enfim, para a realização do trabalho experimental foram construídos, instalados e monitorados, quatro reatores de $20 \mathrm{~L}$ de capacidade unitária, com adaptação feita em depósitos de plástico rígido, conforme mostrado na Figura 1.

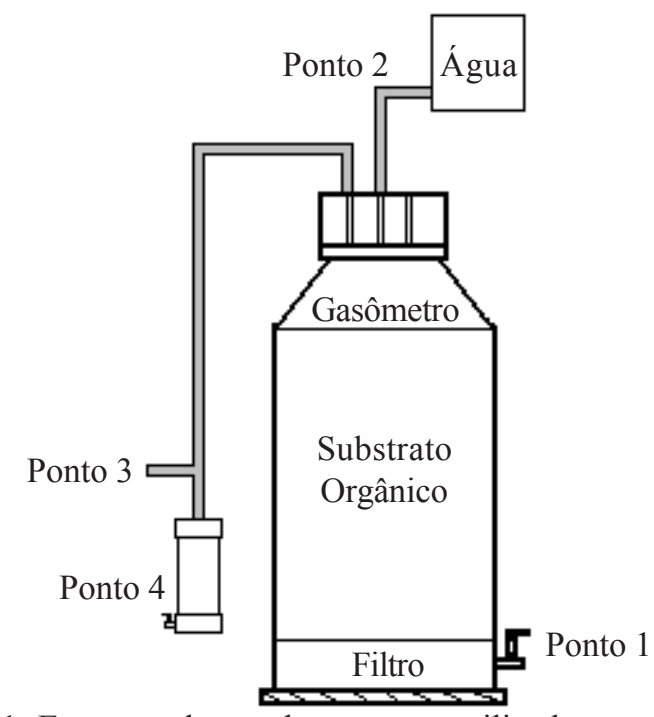

Figura 1. Esquema de um dos reatores utilizados no trabalho experimental

Em cada um dos reatores foram instalados os seguintes dispositivos:

Ponto 1: torneira para coleta do percolado

Ponto 2: distribuidor de água adicionada ao interior do reator

Ponto 3: coleta de biogás para análise

Ponto 4: registrador quantitativo do biogás produzido

Na Tabela 1 são apresentados os parâmetros operacionais aplicados aos reatores constituintes do sistema experimental.

Tabela 1. Parâmetros operacionais aplicados aos reatores

\begin{tabular}{|c|c|c|c|c|}
\hline \multirow{2}{*}{ Reator } & \multirow{2}{*}{$\begin{array}{l}\text { Proporção - \% } \\
\text { Resíduo/Rúmen }\end{array}$} & \multicolumn{2}{|c|}{ Massa - kg } & \multirow{2}{*}{$\begin{array}{l}\mathrm{COA}^{*} \\
\mathrm{~kg} \mathrm{~m}^{-3}\end{array}$} \\
\hline & & Resíduo & Rúmen & \\
\hline A & $100 / 0$ & 8,50 & 0,00 & 425 \\
\hline B & $95 / 5$ & 8,08 & 0,42 & 425 \\
\hline $\mathrm{C}$ & $90 / 10$ & 7,65 & 0,85 & 425 \\
\hline $\mathrm{D}$ & $85 / 15$ & 7,23 & 1,27 & 425 \\
\hline
\end{tabular}

* COA = carga orgânica aplicada

A monitoração do sistema experimental foi realizada nas frações sólida, líquida e gasosa. As análises físicas e químicas empregadas ao longo do período de monitoração do sistema experimental, foram: sólidos totais (ST), sólidos totais voláteis (STV), sólidos totais fixos (STF), carbono orgânico total (COT), nitrogênio total Kjeldhal (NTK), fósforo total (PT) e demanda química de oxigênio (DQO). Todas as análises foram realizadas utilizando-se os métodos preconizados por APHA (1995).

O sistema experimental foi monitorado durante 365 dias, após o qual o material remanescente e parcialmente bioestabilizado em cada um dos reatores, foi caracterizado com a finalidade de se determinar a eficiência de redução dos parâmetros STV, DQO, NTK e PT. 
Com os dados experimentais do trabalho e o programa computacional Table Curve advindo de Jandel Scientific, ajustaram-se modelos matemáticos para se estimar os quantitativos de redução das DQO, NTK, PT e STV, em função do percentual de inóculo utilizado.

\section{RESULTADOS E DISCUSSÃO}

Os resultados da caracterização física e química dos substratos orgânicos aplicados aos reatores, são apresentados na Tabela 2.

Analisando-se os dados da Tabela 2, observa-se que a relação $\mathrm{C} / \mathrm{N}$ da massa "in natura" alimentada aos reatores, situou-se acima da média recomendada para o processo de bioestabilização da matéria orgânica, que é de 20 a 30 e, embora se trabalhando com relações $\mathrm{C} / \mathrm{N}$ variando de 35,83 a 42,18 , não se constatou evidência de desequilíbrio no processo, causado por deficiência de nitrogênio. Observou-se, ainda, que o rúmen contribuiu significativamente para redução da relação $\mathrm{C} / \mathrm{N}$ do substrato.

Na Tabela 3 são apresentados os modelos matemáticos que estimam os quantitativos de redução das DQO, NTK, PT e STV, em função do percentual de inóculo utilizado.

Constata-se na Tabela 3, que o percentual de inóculo utilizado na preparação do substrato apresentou influência significativa na redução dos parâmetros monitorados, visto que os valores dos coeficientes de determinação $\left(\mathrm{R}^{2}\right)$ dos modelos que estimam a taxa de redução em função do percentual de inoculo, foi 0,991 para a DQO, 0,989 para o NTK e 0,914 para os STV. O modelo que estima a redução de PT em função do percentual de inóculo, apresentou o menor coeficiente de determinação, cujo valor foi de 0,841 .

A biodecomposição do substrato orgânico em reator anaeróbio de batelada, é caracterizada por uma fase lenta, que é o período necessário para adaptação e início da multiplicação da massa bacteriana, além de uma fase de decomposição rápida, cujo crescimento bacteriano é máximo. Portanto, a taxa de biodegradação do substrato pode ser descrita por modelos exponenciais.

Segundo Pavlostalhis \& Giraldo-Gomes (1991) apud Sanders et al. (2000) muitos autores descrevem o comportamento da hidrólise da matéria orgânica obedecendo à cinética de $1^{\mathrm{a}}$ ordem, tomando-se como base a biodegradabilidade do substrato a $\mathrm{pH}$ e temperatura constantes, conforme descrito na Eq. 1.

$$
\mathrm{S}=\mathrm{S}_{0} \mathrm{e}^{-\mathrm{kt}}
$$

donde:

$\mathrm{S}$ - massa do substrato no tempo $\mathrm{t}(\mathrm{kg})$

$\mathrm{S}_{0} \quad$ - massa inicial do substrato $(\mathrm{kg})$

$\mathrm{k} \quad$ - constante de biodecomposição do substrato $\left(\mathrm{d}^{-1}\right)$

t $\quad$ - tempo (d)

O parâmetro cinético utilizado para avaliar a influência do inóculo durante o trabalho experimental, foi a constante de biodecomposição do substrato, cujos valores indicam a velocidade do processo, através da Eq. 1, enquanto as equações exponenciais ajustadas com os dados obtidos durante o trabalho experimental, são apresentadas na Tabela 4.

Analisando-se os dados apresentados na Tabela 4, observa-se que as maiores constantes de biodecomposição foram obtidas para o substrato tratado no reator $\mathrm{D}$ ( $15 \%$ de rúmen bovino) evidenciando, assim, a influência do inóculo utilizado na preparação do substrato.

A constante média de biodecomposição determinada para os sólidos totais voláteis neste trabalho, foi de $1,6 \times 10^{-3} \mathrm{~d}^{-1}$. Leite (1997) estudando a biodigestão anaeróbia de resíduos sólidos urbanos inoculados com lodo de esgoto industrial,

Tabela 2. Caracterização física e química dos substratos aplicados aos reatores

\begin{tabular}{|c|c|c|c|c|c|c|c|c|c|}
\hline \multirow{3}{*}{ Reator } & \multicolumn{9}{|c|}{ Parâmetro* } \\
\hline & TU & ST & STF & STV & COT & NTK & $\mathrm{PT}$ & DQO & \multirow{2}{*}{ Relação C/N } \\
\hline & \multicolumn{8}{|c|}{$\%$} & \\
\hline A & 80,83 & 19,17 & 14,97 & 85,03 & 47,24 & 1,12 & 0,24 & 37,28 & 42,18 \\
\hline B & 80,37 & 19,67 & 14,25 & 85,75 & 47,64 & 1,20 & 0,21 & 41,10 & 39,70 \\
\hline $\mathrm{C}$ & 81,82 & 18,18 & 14,21 & 85,79 & 47,66 & 1,26 & 0,27 & 39,95 & 37,83 \\
\hline $\mathrm{D}$ & 81,06 & 18,94 & 15,91 & 84,09 & 46,72 & 1,32 & 0,27 & 41,10 & 35,83 \\
\hline
\end{tabular}

Tabela 3. Modelos matemáticos obtidos para a redução de DQO, NTK, PT e STV, em função do percentual de inóculo utilizado na preparação do substrato

\begin{tabular}{|c|c|c|c|c|c|}
\hline Parâmetro & Modelo* & $\mathrm{R}^{2}$ & $\mathrm{a}$ & $\mathrm{b}$ & $\mathrm{c}$ \\
\hline DQO & $\% \mathrm{DQO}_{\mathrm{Red}}=\mathrm{a}+\mathrm{bx}+\mathrm{cx}^{2,5}$ & 0,991 & 71,032206 & 1,0777138 & 0,00068333591 \\
\hline NTK & $\% \mathrm{NTK}_{\mathrm{Red}}=\mathrm{a}+\mathrm{bx}^{1,5}$ & 0,989 & 68,40021 & 0,41764173 & -- \\
\hline PT & $\% \mathrm{PT}_{\text {Red }}=\mathrm{a}+\mathrm{bx}+\mathrm{cx}^{1,5}$ & 0,841 & 77,759222 & 0,17322045 & 0,300467 \\
\hline STV & $\% \mathrm{STV}_{\mathrm{Red}}=\mathrm{a}+\mathrm{bx}^{1,5}$ & 0,914 & 31,031912 & 0,44829841 & -- \\
\hline
\end{tabular}

Tabela 4. Equações exponenciais ajustadas aos dados experimentais

\begin{tabular}{|c|c|c|c|c|}
\hline Reator & DQO & NTK & PT & STV \\
\hline A & $\mathrm{DQO}=633,7 \cdot \mathrm{e}^{-0,003423942 . t}$ & $\mathrm{NTK}=18,2 \cdot \mathrm{e}^{-0,00318070 . t}$ & $\mathrm{PT}=3,91 \cdot \mathrm{e}^{-0,00426480 . \mathrm{t}}$ & $\mathrm{STV}=1385,5 \cdot \mathrm{e}^{-0,00117893 . \mathrm{t}}$ \\
\hline B & DQO $=698,7 \cdot \mathrm{e}^{-0,003867676 . t}$ & $\mathrm{NTK}=20,1 \cdot \mathrm{e}^{-0,00365209 . t}$ & $\mathrm{PT}=3,51 \cdot \mathrm{e}^{-0,00413365 \cdot \mathrm{t}}$ & $\mathrm{STV}=1433,7 \cdot \mathrm{e}^{-0,00104376 . \mathrm{t}}$ \\
\hline $\mathrm{C}$ & DQO $=679,2 \cdot \mathrm{e}^{-0,004811780 . t}$ & $\mathrm{NTK}=19,5 \cdot \mathrm{e}^{-0,00440942 . \mathrm{t}}$ & $\mathrm{PT}=4,17 \cdot \mathrm{e}^{-0,00758114 . t}$ & $\mathrm{STV}=1325,7 \cdot \mathrm{e}^{-0,00160332 . t}$ \\
\hline $\mathrm{D}$ & DQO $=698,7 \cdot \mathrm{e}^{-0,005713700 \cdot t}$ & $\mathrm{NTK}=21,3 \cdot \mathrm{e}^{-0,00745818 . \mathrm{t}}$ & $\mathrm{PT}=4,35 \cdot \mathrm{e}^{-0,00884030 . \mathrm{t}}$ & $\mathrm{STV}=1353,8 \cdot \mathrm{e}^{-0,00240332 . t}$ \\
\hline
\end{tabular}

DQO - Demanda Química de Oxigênio; NTK - Nitrogênio Total Kjedhal; PT - Fósforo Total; STV - Sólidos Totais Voláteis 
obteve uma constante de bioestabilização média da ordem de $8,7 \times 10^{-4}\left(\mathrm{~d}^{-1}\right)$ para os sólidos totais voláteis.

$\mathrm{Na}$ Figura 2 apresenta-se o comportamento real e o simulado da eficiência de redução de DQO, em função do percentual de inóculo utilizado.

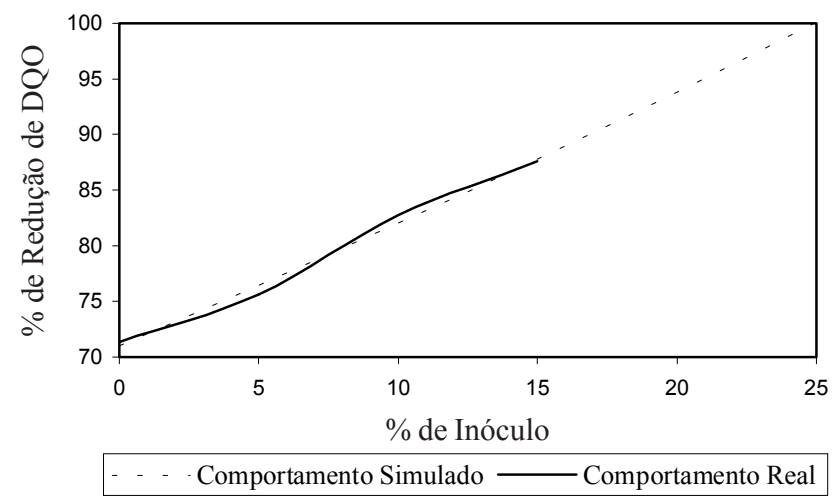

Figura 2. Comportamento real e simulado da eficiência de redução de DQO

Com base no comportamento da Figura 2, observa-se que, para um período de 365 dias e aplicação de $25 \%$ de inóculo, teoricamente haveria a redução de $100 \%$ da DQO aplicada. O comportamento da evolução temporal da massa de DQO no sistema experimental, é mostrado na Figura 3.

Conforme a Figura 3, observa-se que o reator D apresentava uma massa de DQO em torno de $700 \mathrm{~g}$ no início do período de monitoração, enquanto no reator A a massa de DQO era de aproximadamente $630 \mathrm{~g}$. No final do período de monitoração o reator D foi o que apresentou a menor massa de DQO, cerca de $100 \mathrm{~g}$, o que corresponde a uma eficiência de redução de DQO de $86 \%$ para este reator, enquanto nos reatores A, B e $\mathrm{C}$ as eficiências de reduções foram de $81 \%, 73 \%$ e $70 \%$, respectivamente.

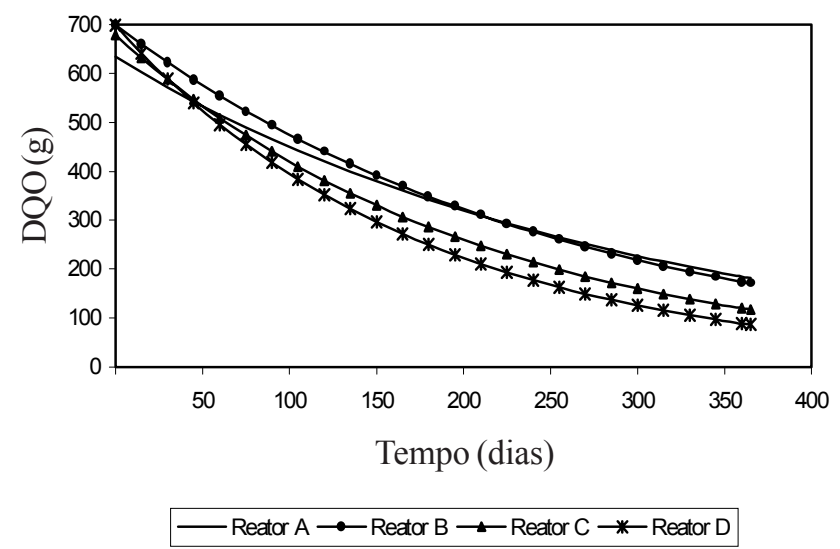

Figura 3. Comportamento da evolução temporal da massa de DQO

Na Figura 4 observa-se o comportamento real e simulado da eficiência de redução de NTK, em função do percentual de inóculo aplicado.

Analisando-se o comportamento da Figura 4, constata-se que, para o tempo de operação de 365 dias, o modelo real demonstra que, com 15\% (percentagem em peso) de inóculo, a massa de NTK seria reduzida em cerca de $93 \%$; em relação ao modelo simulado,

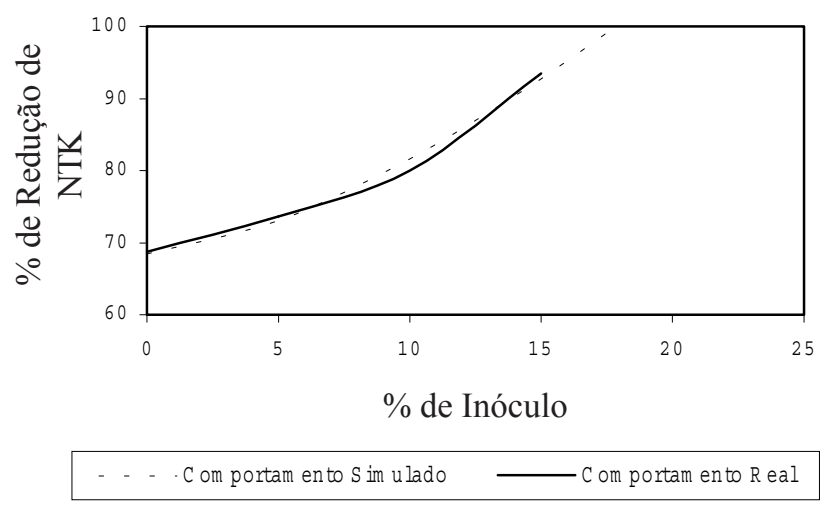

Figura 4. Comportamento real e simulado da eficiência de redução de NTK

ficou denotado que, com 18\% (percentagem em peso) de inóculo, a massa de NTK seria reduzida completamente no mesmo período de tempo, ou seja, 365 dias. Na Figura 5 tem-se o comportamento da evolução temporal da massa de NTK, ao longo do período de monitoração do sistema experimental.

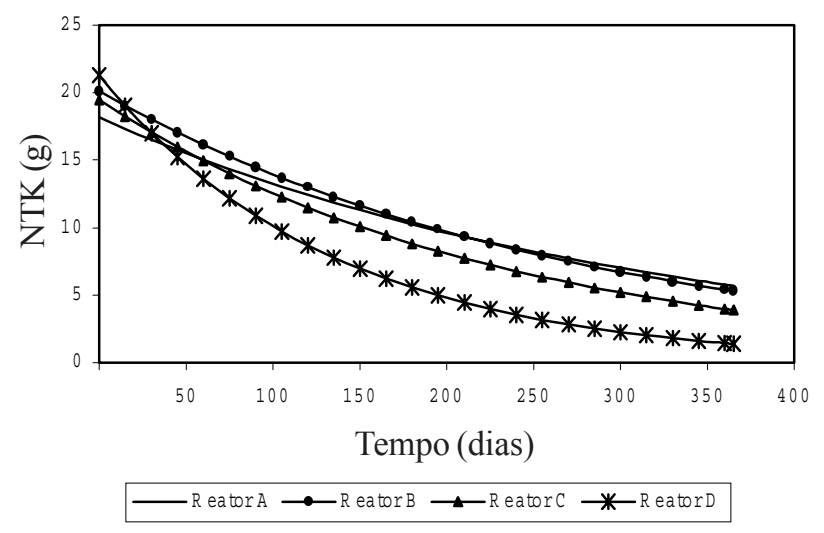

Figura 5. Comportamento da evolução temporal da massa de NTK

Conforme o comportamento da Figura 5, apesar do reator D ter sido alimentado com uma massa de NTK superior à dos demais reatores, no final do período de monitoração este reator apresentou a maior eficiência de redução da massa de NTK.

O comportamento real e estimado da eficiência de redução de fósforo total em função do percentual de inoculo, é mostrado na Figura 6.

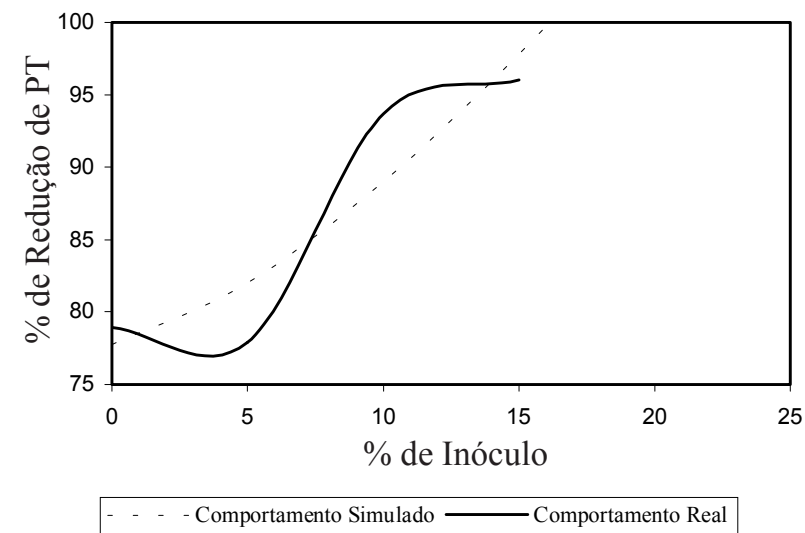

Figura 6. Comportamento real e simulado da eficiência de redução de PT 
Segundo a Figura 6, a aplicação de aproximadamente $16 \%$ de rúmen bovino teoricamente conduziria à redução de $100 \%$ da massa de PT aplicada. Observa-se, ainda, que o comportamento real apresentou discrepâncias com relação ao modelo estimado, o que se justifica pelo baixo coeficiente de determinação para a redução de PT apresentado anteriormente na Tabela 3.

As altas reduções de NTK e PT se devem às reações de hidrólise que ocorrem nas primeiras fases do processo de bioestabilização anaeróbia, na qual uma grande quantidade desses nutrientes é solubilizada e lixiviada do interior dos reatores, através dos líquidos percolados. Na Tabela 5, tem-se os dados advindos da aplicação do balanço de massa para NTK e PT.

Verificando-se os dados apresentados na Tabela 5, observa-se que as maiores quantidades de NTK e PT foram hidrolisadas e liberadas do reator, através dos líquidos percolados.

Tabela 5. Balanços de massa para NTK e PT

\begin{tabular}{|c|c|c|c|c|c|c|c|c|}
\hline \multirow{2}{*}{ Reator } & \multicolumn{4}{|c|}{ NTK (\%) } & \multicolumn{4}{|c|}{ PT (\%) } \\
\hline & $\mathrm{M}_{\mathrm{AF}}$ & $\mathrm{M}_{\mathrm{AC}}$ & $\mathrm{M}_{\mathrm{EP}}$ & $\mathrm{M}_{\mathrm{EB}}$ & $\mathrm{M}_{\mathrm{AF}}$ & $\mathrm{M}_{\mathrm{AC}}$ & $\mathrm{M}_{\mathrm{EP}}$ & $\mathrm{M}_{\mathrm{EB}}$ \\
\hline A & 100 & 31,32 & 60,44 & - & 100 & 21,08 & 78,92 & - \\
\hline B & 100 & 26,37 & 65,67 & - & 100 & 22,12 & 77,88 & - \\
\hline $\mathrm{C}$ & 100 & 20,00 & 73,33 & - & 100 & 6,28 & 93,72 & - \\
\hline D & 100 & 6,57 & 85,92 & - & 100 & 3,97 & 96,03 & - \\
\hline
\end{tabular}

$\mathrm{M}_{\mathrm{AF}}=$ massa afluente; $\mathrm{M}_{\mathrm{EP}}=$ massa liberada através do percolado; $\mathrm{M}_{\mathrm{AC}}=$ massa acumulada no reator; $\mathrm{M}_{\mathrm{EB}}=$ massa convertida em biogás

A Figura 7 mostra o comportamento real e simulado da eficiência de redução de sólidos totais voláteis, em função do percentual de inóculo utilizado na preparação do substrato.

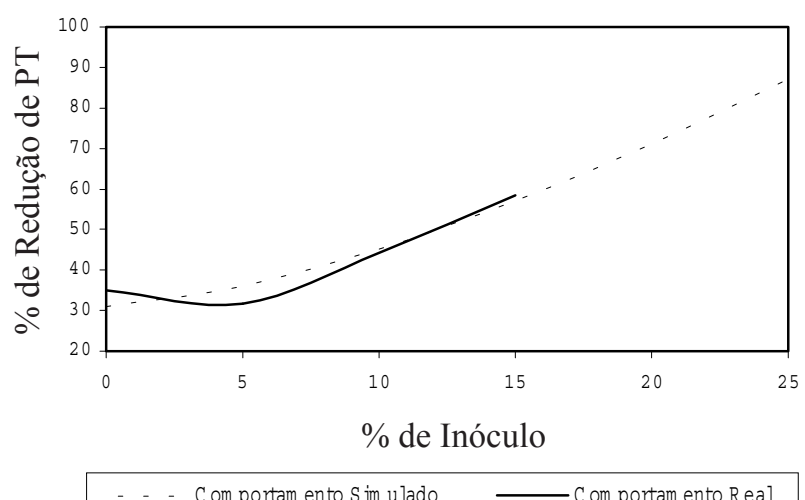

Figura 7. Comportamento real e estimado da eficiência de redução de STV

Reportando-se aos STV apresentados na Figura 7, constata-se que a eficiência real de redução de STV atingiu o patamar médio de $58 \%$ para o tempo de operação de 365 dias. Para o modelo simulado, constatou-se que, mesmo se trabalhando com $25 \%$ de inóculo, a eficiência de redução de STV ficaria em torno de $84 \%$, para o período de operação de 365 dias.

\section{CONCLUSÕES}

1. A eficiência do processo de bioestabilização anaeróbia, no caso específico deste trabalho, foi função do percentual de inóculo aplicado.

2. Dentre os percentuais de inóculo aplicados, o percentual de $15 \%$ contribuiu mais significativamente para o desempenho do processo, haja vista, formar substrato com uma relação $\mathrm{C} / \mathrm{N}$ equilibrada, além de proporcionar maior massa de microrganismos.

3. O processo de tratamento anaeróbio de resíduos sólidos orgânicos com alta concentração de sólidos apresenta possibilidade real de se tornar alternativa promissora de tratamento, para esses tipos de resíduos.

\section{AGRADECIMENTOS}

Os autores agradecem ao Conselho Nacional de Desenvolvimento Científico e Tecnológico (CNPq), o apoio financeiro concedido para a realização deste trabalho.

\section{LITERATURA CITADA}

APHA American Public Health Association. Standard methods for examination of the water and wastewater. $19^{\text {th }}$ ed. Washington: APHA, 1134p. 1995.

IPT. Instituto de Pesquisa Tecnológica. Lixo Municipal: Manual de gerenciamento integrado. IPT/CEMPRE, São Paulo, 2000. 180p.

Leite, V.D. Origem e composição dos resíduos sólidos. Trabalho apresentado à comissão julgadora do concurso público para Professor Titular da UEPB (Universidade Estadual da Paraíba). Campina Grande, 1998. 78p.

Leite, V.D. Processo de tratamento anaeróbio de resíduos sólidos urbanos inoculados com lodo de esgoto industrial. São Carlos: EESC/USP. 1997. 250p. Tese Doutorado

Metcalf \& Eddy. Waste engineering: Treatment, disposal, refuse. $3^{\text {rd }}$ Ed. McGraw - Hill International Edition, 1991. 1334p.

Polprasert, C. Organic waste recycling. Bangkok: Environmental engineering division, Asian Institute of Technology. 1989. 357p.

Sanders, M.; Geerink, M.; Zeeman, G.; Lettinga, G. Anaerobic hydrolysis of particulate substrates. Water Science and Technology, London, v.41, n.3, p.17-24, 2000. 